\title{
Deficiency of IKKe inhibits inflammation and induces cardiac protection in high-fat diet-induced obesity in mice
}

\author{
CHANGCHUN CAO ${ }^{2 *}$, LIANGPENG LI $^{1 *}$, WEN CHEN $^{1}$, YIFAN ZHU $^{1}$, \\ YONGCHAO QI ${ }^{1}, \mathrm{XIAODI} \mathrm{WANG}^{1}, \mathrm{XIN} \mathrm{WAN}^{1}$ and XIN CHEN ${ }^{1}$ \\ Departments of ${ }^{1}$ Thoracic and Cardiovascular Surgery, ${ }^{2}$ Nephrology, Nanjing Hospital Affiliated to \\ Nanjing Medical University, Nanjing, Jiangsu 210006, P.R. China
}

Received December 17, 2013; Accepted April 9, 2014

DOI: $10.3892 /$ ijmm.2014.1746

\begin{abstract}
Immune response and metabolic regulation have been recognized as a central homeostatic mechanism, the dysfunction of which can trigger a cluster of chronic metabolic disorders, particularly obesity, type II diabetes and cardiovascular disease. Serine/threonine kinase I $\kappa$ B kinase (IKK) $\varepsilon$ is a multifunctional regulator that participates in immune regulation, cell proliferation and transformation, and oncogenesis. In the present study, we investigated the role of IKKe in cardiovascular disorders using murine models of apolipoprotein E-deficient $[\mathrm{ApoE}(-/-)]$ mice and ApoE/IKKe double-knockout [ApoE(-/-)/IKKe(-/-)]mice, which were fed a normal diet (ND) and high-fat diet (HFD) for 12 weeks, respectively. Results of this study showed that mouse obesity correlated in vivo with an increased expression of IKKe. Additionally, chronic low-grade inflammation in cardiac tissue was evident in ApoE(-/-) mice, but was markedly reduced in $\operatorname{ApoE}(-/-) /$ $\operatorname{IKK\varepsilon }(-/-)$ mice. However, serum lipid levels in the ApoE(-/-) mice group were not significantly higher than those of the ApoE(-/-)/IKKe(-/-) group. Furthermore, immunofluorescence and western blot analysis demonstrated evident increases in the expression of nuclear factor- $\kappa \mathrm{B}(\mathrm{NF}-\kappa \mathrm{B})$ pathway components and downstream factors in the ApoE(-/-) mice group, while these increases were blocked in the $\mathrm{ApoE}(-/-) / \operatorname{IKK} \varepsilon(-/-)$ group. Taken together, these data indicate that deficiency of IKKE prevented obesity and inflammatory response in the murine hearts in ApoE(-/-) and ApoE(-/-)/IKKe(-/-) mice fed an ND and HFD, respectively, suggesting that IKKe may play a role in HFD-induced inflammation in hearts of obese mice
\end{abstract}

Correspondence to: Professor Xin Chen, Department of Thoracic and Cardiovascular Surgery, Nanjing Hospital Affiliated to Nanjing Medical University, 68 Changle Road, Nanjing, Jiangsu 210006, P.R. China

E-mail: stevecx@njmu.edu.cn

${ }^{*}$ Contributed equally

Key words: ІкВ kinase $\varepsilon$, nuclear factor- $\kappa \mathrm{B}$, diet, obesity, inflammation and may serve as a novel target for the treatment of a variety of metabolism-associated cardiovascular diseases.

\section{Introduction}

The incidence of obesity worldwide has increased significantly during recent decades. Consequently, obesity and its associated disorders constitute a serious threat to the current and future health of populations (1). It is now well established that obesity results in a state of chronic low-grade inflammation thought to contribute to several metabolic disorders, including insulin resistance, type II diabetes and cardiovascular disease $(2,3)$. In individuals with these metabolic disorders, different cardiovascular disorders often develop simultaneously, thus obese patients present with an increased risk of suffering from insulin resistance and type II diabetes, these being frequently associated with cardiovascular disease including hypertension, atherosclerosis, arrhythmias and heart failure, suggesting the sharing of similar pathogenic mechanisms $(4,5)$. Nevertheless, the exact underlying mechanisms involved remains elusive.

Transcription factor nuclear factor- $\kappa \mathrm{B}(\mathrm{NF}-\kappa \mathrm{B})$ is a potent transcriptional activator and plays an important role in a variety of physiological and disease processes, including immune and inflammatory responses, cardiovascular diseases, insulin resistance and type II diabetes (6-8). The NF- $\kappa$ B proteins are a family of ubiquitously expressed transcription factors that, in mammals, comprise five members: NF- $\mathrm{B} 1$ ( $\mathrm{p} 50$; precursor protein: p105), NF-кB2 (p52; precursor protein: p100), p65 (RelA), c-Rel (Rel) and RelB, which share the so-called Rel homology domain that mediates DNA binding, dimerization and nuclear translocation $(9,10)$. NF- $\kappa \mathrm{B}$ activation is triggered by the phosphorylation of the regulatory protein $\mathrm{I} \kappa \mathrm{B}$, and regulated through the canonical $\mathrm{I} \kappa \mathrm{B}$ kinases [kinase $\mathrm{I} \kappa \mathrm{B}$ kinase (IKK) $\alpha$, IKK $\beta$ ] and non-canonical IKK-related kinases (IKKع, TBK1). Findings of previous studies suggested a role for IKK $\alpha$ and $\beta$ in NF- $\kappa B$ activation that leads to an increase in transcription of inflammation, and cell growth/survival of genes (11-13). However, the kinase domain of IKKe only exhibits 27 and $24 \%$ identity to IKK $\alpha$ and $\beta$, respectively (14), suggesting that IKK $\varepsilon$ may have a different role to IKK $\alpha$ and $\beta$.

The stress-activated protein kinase IKKe is a central signal transducer that regulates immune response, cell proliferation and transformation, and oncogenesis (15-20). Results 
of a previous study showed that a high-fat diet (HFD) can increase $\mathrm{NF}-\kappa \mathrm{B}$ activation in mice, which leads to a sustained elevation of IKKe levels in the liver, adipocytes and adipose tissue macrophages. Deletion of the IKKe gene rendered mice partially resistant to the HFD-dependent development of obesity, insulin sensitivity, hepatic steatosis and inflammation (15). Based on this established connection between obesity-induced insulin resistance and enhanced $\mathrm{NF}-\kappa \mathrm{B}$ activity in insulin target tissues, we investigated whether IKKe has a similar role in HFD-induced cardiovascular disorders.

To address this issue, murine models of apolipoprotein E-deficient $[\mathrm{ApoE}(-/-)]$ mice and ApoE/IKKe double-knockout mice $[\mathrm{ApoE}(-/-) / \mathrm{IKK} \varepsilon(-/-)]$ were established. One group of ApoE(-/-)/IKKe(-/-) mice was switched from normal diet (ND) to HFD for 12 weeks from 8 weeks of age, respectively, and another control group [ApoE(-/-) mice] also maintained the same diet. Therefore, IKKe modulates HFD-induced obesity, inflammation by activation of $\mathrm{NF}-\kappa \mathrm{B}$-dependent gene transcription and may be useful as a therapeutic target in the treatment of metabolism-associated cardiovascular diseases.

\section{Materials and methods}

Animal models. IKKe knockout $\left(\right.$ B6.Cg-Ikbke $\left.<^{\mathrm{tm}^{\mathrm{Tman}}}>/ \mathrm{J}\right)$ mice, purchased from Jackson Laboratory (Bar Harbor, ME, USA), underwent rederivation to achieve pathogen-free status in the Model Animal Research Center of Nanjing University (Nanjing, China). ApoE knockout [ApoE(-/-)] mice were obtained from the Model Animal Research Center of Nanjing University at the age of 8 weeks. IKKe knockout mice were bred into the ApoE knockout genetic background to obtain the $\operatorname{ApoE}(-/-) / \operatorname{IKK} \varepsilon(-/-)$ group of mice, as reported previously (21). At the age of 8 weeks, the male $\operatorname{ApoE}(-/-)$ and ApoE(-/-)/IKKe(-/-) mice were placed on a HFD containing $60 \%$ calories of lipid (soybean oil, 5.5\%; lard, 54.5\%; D12492; Research Diets, Inc., New Brunswick, NJ, USA) and fed for 12 weeks, whereas the remaining mice were kept as controls on a standard ND containing $10 \%$ calories for 12 weeks. All the mice were housed in specific pathogen-free box cages at a constant temperature of $23 \pm 2{ }^{\circ} \mathrm{C}$ and humidity of $60 \pm 10 \%$. An inverted light-dark cycle of 12:12 h was used and the animals had free access to food and water. Both male and female mice were 12 weeks old at the time of experiments. Animal experiments were performed in compliance with the Institute of Laboratory Animal Research Guide for the Care and Use of Laboratory Animals of the National Institutes of Health and approved by the Institutional Animal Care and Use Committee of Nanjing Medical University.

Plasma parameters. After being fed with ND and HFD for 12 weeks, respectively, mice were fasted for $12 \mathrm{~h}$ prior to being anesthetized through administration of intraperitoneal injection of pentobarbital ( $50 \mathrm{mg} / \mathrm{kg}$ body weight), and the adequacy of anesthesia was evaluated by monitoring hind limb reflexes. Blood samples were obtained from the retro-orbital plexus. Total cholesterol (TC), triglycerides (TG), low-density lipoprotein (LDL) and high-density lipoprotein (HDL) in the serum were determined using colorimetric enzymatic assays that were adjusted to the 96-well format (Sigma, St. Louis, MO, USA).
Tissue collection and histological analysis. After euthanasia, murine heart tissues were collected, fixed in $4 \%$ formalin for $48 \mathrm{~h}$ and then embedded in paraffin. Serial aortic sections $(4 \mu \mathrm{m})$ were then prepared and stained with hematoxylin and eosin (H\&E) for histopathology. Formalin-fixed, paraffin-embedded sections were subjected to antigen retrieval by boiling in $0.01 \mathrm{M}$ sodium citrate buffer ( $\mathrm{pH}$ 6.0) in a microwave oven after dewaxing and rehydration. The sections were treated with $3 \%$ hydrogen peroxide for $15 \mathrm{~min}$ to block endogenous peroxidase activity and incubated in buffered normal horse serum to prevent non-specific binding of antibodies. The sections were then incubated separately for $14 \mathrm{~h}$ with antibodies against tumor necrosis factor- $\alpha$ (TNF- $\alpha)$ (1:800), macrophage-colony stimulating factor (M-CSF) (1:200), CD4 T cells (CD4) (1:200) (all from Abcam, Cambridge, MA, USA), IKKe (1:500; Novus Biologicals, Littleton, CO, USA) followed by incubation with horseradish peroxidase (HRP)-conjugated goat anti-rabbit IgG (Beijing Zhongshan Golden Bridge Biotechnology Co., Ltd., Beijing, China) for $1 \mathrm{~h}$ at $37^{\circ} \mathrm{C}$ in a humidified box. The signal of each antibody was developed using the substrate diaminobenzidine (DAB; Beijing Zhongshan Golden Bridge Biotechnology Co.). Sections were counterstained with hematoxylin and photomicrographs were obtained using an Olympus BX-URA2 camera.

Quantitative reverse transcription PCR ( $q R T-P C R)$. For qPCR, total RNA was extracted from the frozen mouse tissue using TRIzol (Invitrogen, Carlsbad, CA, USA) and reverse-transcribed into cDNA using oligo(dT) primers with a transcriptor first-strand cDNA synthesis kit. PCR amplifications were quantified using the SYBR-Green PCR Master mix (Applied Biosystems, Foster City, CA, USA) and normalized to GAPDH gene expression. The primers for quantitative PCR are shown in Table I.

Western blot analysis. Samples of total protein $(50 \mu \mathrm{g})$ were extracted from the murine heart of each group and separated by SDS-PAGE. Proteins were then transferred to a polyvinylidene fluoride (PVDF) membrane (Millipore Corp., Billerica, MA, USA), washed with a dilution of 1:1,000 in TBS with Tween (TBST; Promega Corp., Madison, WI, USA) twice (10 min per wash) and blocked using 5\% non-fat milk powder for $1 \mathrm{~h}$. The membranes were then probed with the following primary antibodies in TBS with Tween plus 5\% milk overnight at $4^{\circ} \mathrm{C}$ : anti-IKKe (1:200; Novus Biologicals), anti-T-p50 (1:200), anti-T-p65 (1:200), anti-IкB- $\alpha$ (1:200), anti-Pi-50 (1:300), anti-Pi-65 (1:300), anti-IL-1 $\beta$ (1:500), anti-TGF- $\beta$ (1:500), and anti-GAPDH (1:5,000) (all from Santa Cruz Biotechnology, Inc., Santa Cruz, CA, USA). The following day, the PVDF membranes were washed with TBST four times (10 min per wash) and incubated with appropriately diluted peroxidase-conjugated secondary antibodies and goat anti-rabbit IgG (anti-mouse and anti-rabbit immunoglobulins, respectively; Santa Cruz Biotechnology, Inc.) at room temperature for $1 \mathrm{~h}$. The membranes were then washed with TBST four times as previously described. Specific proteins were detected using an ECL reagent and captured on Hyperfilm (both from GE Healthcare, Piscataway, NJ, USA). The results were then analyzed through the Quantity One software 
Table I. The primers for vector construct.

\begin{tabular}{lll}
\hline Name & \multicolumn{1}{c}{ Forward } & \multicolumn{1}{c}{ Reverse } \\
\hline IKK $\alpha$ & GTCAGGACCGTGTTCTCAAGG & CAGGCACCGTTCACACATAC \\
IKK $\beta$ & AGCGAACGGTCATCCACGTCTT & ACTGGTGATCTCTATGCTGTCA \\
IKK $\varepsilon$ & ACTCCACTCACGGCAAATTC & GCTTCTTTGATGTTACTGAGGGC \\
TBK1 & CAAGATCCATGTCCAACGTG & AGATGGCAATCGTGTTGTGGGC \\
GAPDH & TTCTGGAAGTCCATACGCATTG & TCTCCATGGTGGTGAAGACA
\end{tabular}

IKK, IкB kinase.

for semiquantification of the mean gray value of each blot. Subsequently, the SPSS statistical software was used to perform one-way analysis of variance (ANOVA) to detect the differences among groups of mice. All presented results are representative of at least three independent experiments.

Immunofluorescence staining. Tissues collected for morphological analysis of the heart were prepared as $4 \mu \mathrm{m}$ thick serial optimum cutting temperature (OCT) compound-embedded cryosections. The sections were fixed in $4 \%$ paraformaldehyde for $30 \mathrm{~min}$, washed in PBS, and incubated in buffered normal goat serum to preclude non-specific binding of antibodies at room temperature for $1 \mathrm{~h}$. The sections were then incubated separately overnight with antibodies against Pi-p50, Pi-p65 (1:100; Santa Cruz Biotechnology, Inc.), respectively, followed by incubation with Alexa Fluor 592 goat anti-rabbit $\operatorname{IgG}\left(1: 200\right.$; Invitrogen) at $37^{\circ} \mathrm{C}$ for $1 \mathrm{~h}$ in a humidified box. Subsequently, the sections were washed in PBS and counterstained with Hoechst DNA dye $(10 \mathrm{mg} / \mathrm{ml}$; Sigma) to irradiate nuclei. Photomicrographs were captured at random using an Olympus BX-URA2 camera in 5 sections per mouse sample.

Immunoprecipitation analysis. For immunoprecipitation analysis, tissue samples were homogenized in ice-cold PBS and lysed for $2 \mathrm{~h}$ at $4^{\circ} \mathrm{C}$ in RIPA buffer (Roche Diagnostics Norge AS, Oslo, Norway). Lysates were cleared by centrifugation at $4^{\circ} \mathrm{C}$ for $10 \mathrm{~min}$ at $15,000 \mathrm{x} \mathrm{g}$. Protein $\mathrm{A} / \mathrm{G}$ magnetic beads (Millipore) were washed and resuspended individually according to the manufacturer's instructions and subsequently incubated overnight at $4^{\circ} \mathrm{C}$ with $10 \mu \mathrm{g}$ anti-IKKe (1:500) or anti-T-p50 (1:500), anti-T-p65 (1:500) (all from Santa Cruz Biotechnology) antibody. Antibody-bound beads were washed three times and incubated with cell lysate samples $(500 \mu \mathrm{g})$ for $2 \mathrm{~h}$ at $4^{\circ} \mathrm{C}$ in a volume of $1 \mathrm{ml}$. After washing, immunoprecipitated proteins were eluted by heating at $100^{\circ} \mathrm{C}$ for $5 \mathrm{~min}$ in Laemmli sample buffer. Samples of equal protein content were separated by SDS-PAGE, transferred to PVDF and subsequently processed according to the procedure described for western blot analysis.

Statistical analysis. The data are presented as the means \pm SEM. Differences among groups were determined by a two-way ANOVA followed by SPSS 17.0 (SPSS, Inc., Chicago, IL, USA) software. Comparisons between two groups were performed using an unpaired Student's t-test. The significance level was set at $\mathrm{P}<0.05$.

\section{Results}

IKKE expression induced by HFD is upregulated in ApoE(-/-) murine hearts. To investigate the potential response of the inducible IKK family members to dietary stresses, we first analyzed the mRNA levels of IKKs after ND or HFD for 12 weeks by qRT-PCR. IKKe mRNA levels in the murine hearts were significantly upregulated at 12 weeks after HFD, compared with the expression of IKK $\alpha, \beta$ and TBK1 (Fig. 1A). Consistent with these data, the protein expression levels of $\mathrm{IKK \varepsilon}$ in hearts from ApoE(-/-) mice after 12-week HFD also showed the same trends by western blot analysis assays (Fig. 1B and C), suggesting that IKKe plays a different role to IKK $\alpha, \beta$ and TBK1 in hearts. We then examined expression in murine hearts by immunohistochemical analysis. As expected, $\mathrm{IKK} \varepsilon$ expression was frequently elevated in the ApoE(-/-) group HFD-fed mice as compared to the ND controls. Notably, we observed distinct IKK $\varepsilon$ staining, distributed diffusely in the nucleus or cytoplasm, or both (Fig. 1D). However, no staining was observed in the ApoE(-//)/IKKe(-/-) group at the same time, confirming that IKK $\varepsilon$ expression was absent in the hearts. Thus, the altered pattern of IKK $\varepsilon$ expression in the hearts, suggested the possibility that IKKع functions as a modulator of HFD-induced metabolic and cardiovascular disorders.

ApoE(-/-)/IKKE(-/-) group mice gain less weight although similar plasma lipid levels are produced by HFD compared to ApoE(-/-)group mice. To examine the potential role of IKK $\varepsilon$ in obesity and lipid metabolism, male ApoE(-/-) mice and $\mathrm{ApoE}(-/-) / \mathrm{IKK} \varepsilon(-/-)$ mice were fed a HFD or a regular ND for 12 weeks starting at the age of 8 weeks, while monitoring body weight and food intake. Body weights were determined every third week during the course of the study. As shown in Fig. 2A (Table II), ApoE(-/-) mice gained almost $20 \mathrm{~g}$ following $\mathrm{HFD}$, whereas the $\mathrm{ApoE}(-/-) / \mathrm{IKK} \varepsilon(-/-)$ group mice gained less weight than the ApoE(-/-) group mice (final weight $34.1 \pm 0.45 \mathrm{~g}$ vs. $39.7 \pm 0.52 \mathrm{~g} ; \mathrm{P}<0.05)$. There was no obvious alteration on food intake, either at the beginning or end of the study (Fig. 2B, Table II), indicating that changes in weight gain could not be attributed to greater food ingestion.

Although no significant serum lipid alterations were observed between the ApoE(-/-) and ApoE(-/-)/IKKe(-/-) murine groups following ND, HFD produced marked increase in the serum TC and TG levels of the ApoE(-/-) and ApoE(-/-)/IKKe(-/-) murine groups. However, after exposure for 12 weeks to HFD, no distinct difference in the level of 
Table II. Metabolic parameters of different groups of mice exposed to a ND or HFD diet.

\begin{tabular}{|c|c|c|c|c|}
\hline \multirow[b]{2}{*}{ Parameter } & \multicolumn{2}{|c|}{ AKO } & \multicolumn{2}{|c|}{ DKO } \\
\hline & ND & HFD & ND & HFD \\
\hline Number & 9 & 11 & 8 & 10 \\
\hline BW at week $0(\mathrm{~g})$ & $21.0 \pm 0.32$ & $20.9 \pm 0.35$ & $21.1 \pm 0.26$ & $20.8 \pm 0.24$ \\
\hline BW at week $12(\mathrm{~g})$ & $28.4 \pm 0.29$ & $39.7 \pm 0.52^{\mathrm{a}}$ & $28.9 \pm 0.47$ & $34.1 \pm 0.45^{\mathrm{b}, \mathrm{c}}$ \\
\hline $\begin{array}{l}\text { Food intake per } \\
\text { BW }(\mathrm{mg} / \mathrm{g})\end{array}$ & $148.5 \pm 3.0$ & $122.8 \pm 3.1$ & $142.6 \pm 3.8$ & $115.3 \pm 3.9$ \\
\hline $\mathrm{TC}(\mathrm{mmol} / \mathrm{l})$ & $10.1 \pm 0.75$ & $22.3 \pm 0.64^{\mathrm{a}}$ & $10.2 \pm 0.31$ & $21.4 \pm 0.71^{\mathrm{b}}$ \\
\hline $\mathrm{TG}(\mathrm{mmol} / \mathrm{l})$ & $1.23 \pm 0.14$ & $3.34 \pm 0.25^{\mathrm{a}}$ & $1.22 \pm 0.11$ & $3.31 \pm 0.16^{\mathrm{b}}$ \\
\hline $\mathrm{LDL}(\mathrm{mmol} / \mathrm{l})$ & $0.96 \pm 0.09$ & $6.22 \pm 0.31^{\mathrm{a}}$ & $1.17 \pm 0.06$ & $6.50 \pm 0.20^{\mathrm{b}}$ \\
\hline HDL (mmol/l) & $1.84 \pm 0.07$ & $0.77 \pm 0.05^{\mathrm{a}}$ & $2.06 \pm 0.10$ & $0.64 \pm 0.04^{\mathrm{b}}$ \\
\hline
\end{tabular}

ND, normal diet; HFD, high-fat diet; BW, body weight; TC, total cholesterol; TG, triglyceride; LDL, low-density lipoprotein; HDL, high-density lipoprotein. Values are means \pm SEM. ${ }^{a} \mathrm{P}<0.05$ vs. AKO ND; ${ }^{b} \mathrm{P}<0.05$ vs. DKO ND; ${ }^{\mathrm{c}} \mathrm{P}<0.05$ vs. AKO HFD.
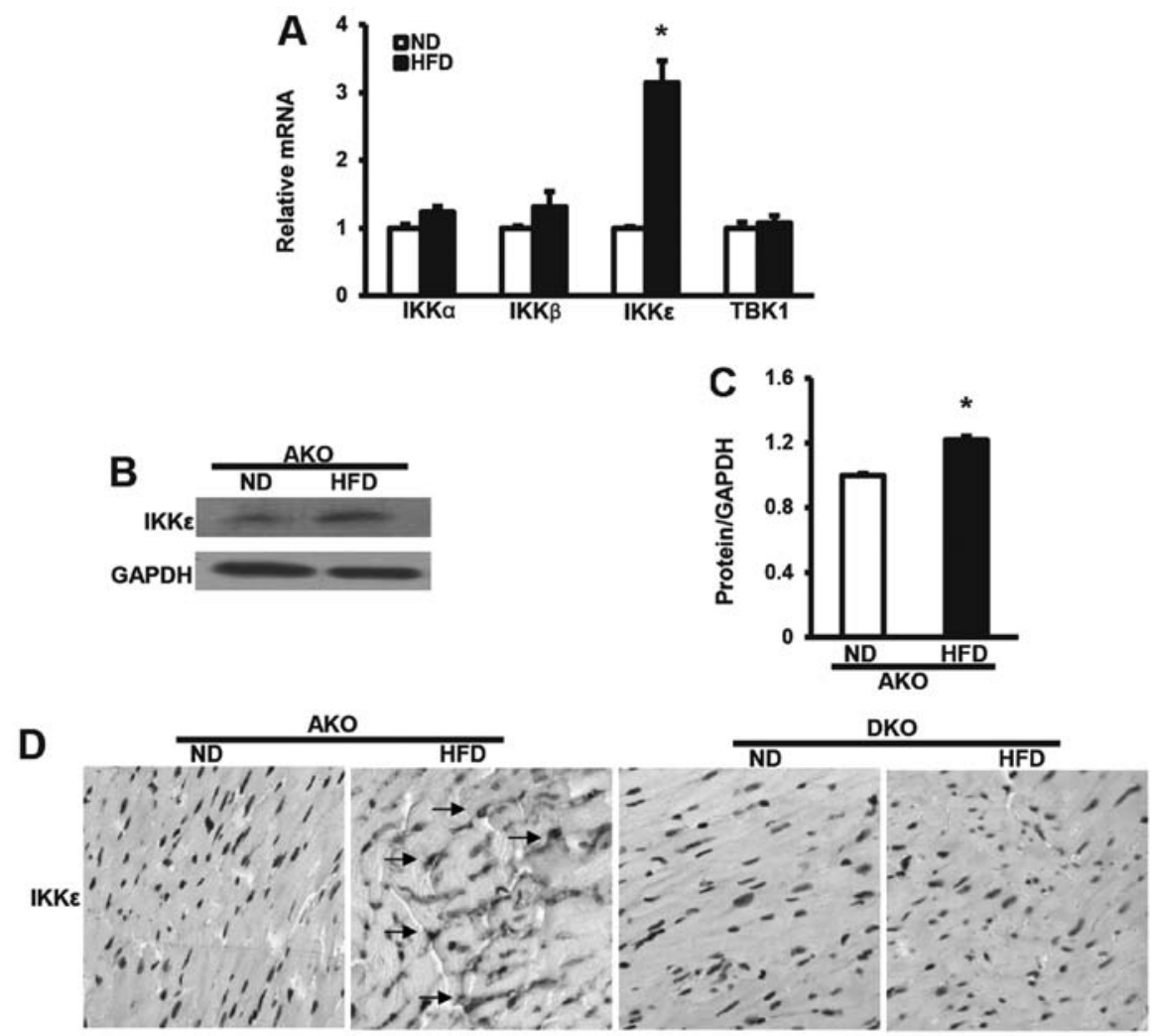

Figure 1. Expression and localization of IKB kinase (IKK) $\varepsilon$ in murine hearts. (A) Quantitative polymerase chain reaction (qPCR) analysis on the expression of genes encoding IKK family members in apolipoprotein E-deficient [ApoE(-/-)] murine hearts. White bars, ApoE(-/-) mice, normal diet (ND); black bars, ApoE(-/-) mice, high-fat diet (HFD) for 12 weeks, $n=4$ per group. Data are presented as the means \pm SEM and the average ND value was set as $1 .{ }^{*} \mathrm{P}<0.05$ vs. AKO ND. (B) IKKe was detected by western blot analysis. GAPDH was used as an internal loading control. (C) Statistical results for ApoE(-/-) (AKO) mice fed with ND (white bars) or HFD (black bars) as indicated, $\mathrm{n}=4$ per group. ${ }^{*} \mathrm{P}<0.05$ vs. AKO ND. (D) Immunohistochemical staining of IKK $\varepsilon$ showing the distribution of $\mathrm{IKK} \varepsilon$ in murine hearts (arrows, $\mathrm{x} 400$ ). Data are presented as the mean \pm SEM and are representative of three independent experiments.

either serum TC or TG was observed between the ApoE(-/-) and ApoE(-/-)/IKKe(-/-) murine groups (Fig. 2C, Table II). Consistent with these data, the LDL measurements also showed the same trends as that of TC and TG, whereas the HDL levels were totally reversed (Fig. 2C, Table II). Collectively, these data suggest that IKKe deficiency leads to decreased weight gain, but not plasma lipid levels.

IKKE deficiency attenuates chronic, high-fat diet-induced inflammation in the heart after HFD feeding. To assess in 

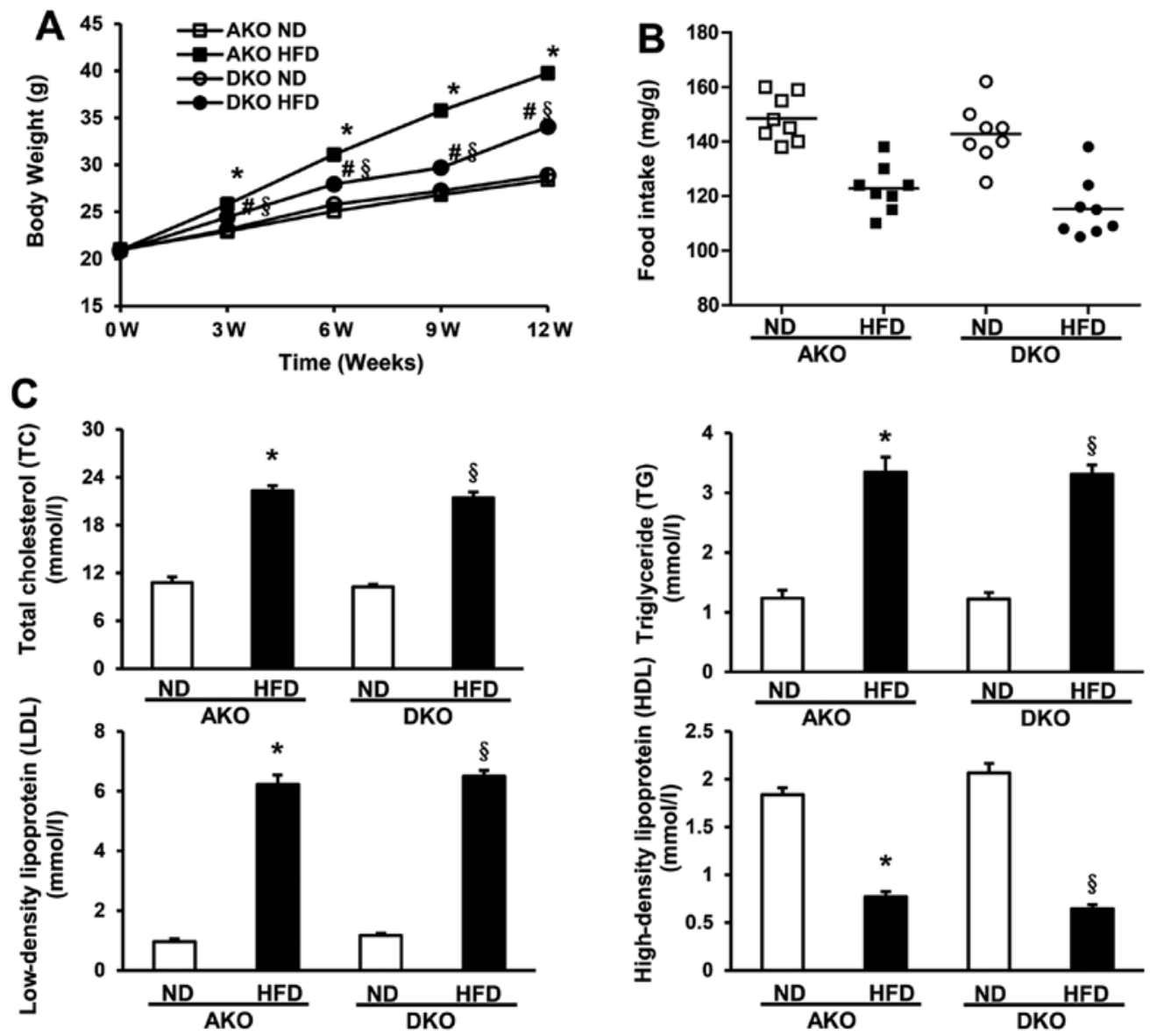

Figure 2. Apolipoprotein E-deficient [ApoE(-/-)]/ IкB kinase (IKK) $\varepsilon(-/-)$ murine group blunts weight gain, however, similar plasma lipid levels were produced by high-fat diet (HFD) compared to ApoE(-/-) murine group. (A) Body weight measured for mice fed with normal diet (ND) or HFD is shown at time-points of 0, 3, 6, 9 and 12 weeks. (B) Food intake per body weight was measured each morning by weighing of ND or HFD, and the mice of each group were measured at three different time-points from week 0, 6-12 to obtain the average food intake, n=8-11 per group. (C) Serum total cholesterol (TC), triglycerides (TG), low-density lipoprotein (LDL) and high-density lipoprotein (HDL) levels analysis after 12 weeks fed with ND or HFD as indicated, n=8-11 per group. *P $<0.05$ vs. AKO ND; ${ }^{\circledR} \mathrm{P}<0.05$ vs. DKO ND; ${ }^{*} \mathrm{P}<0.05$ vs. AKO HFD.

more detail whether the impacts of IKKe deficiency could be seen in the adult mammalian heart, we measured the expression of key metabolic and inflammatory markers followed by histopathological analysis. H\&E staining of myocardial nuclei exhibited slightly but significantly higher heterogeneity, disarray and uneven distribution in the $\operatorname{ApoE}(-/-) / \operatorname{IKK} \varepsilon(-/-)$ group, while no significant changes were detected in the other groups (Fig. 3A). However, Oil Red O staining revealed that lipid deposition was not different between the ApoE(-/-) and ApoE(-/-)/IKKe(-/-) murine groups mice (data not shown).

Comparison of ApoE(-/-) and ApoE(-/-)/IKKe(-/-) animals on ND revealed similar inflammatory mediators including TNF- $\alpha$, CD4 and M-CSF (Fig. 3B). Following HFD feeding, the inflammatory markers were increased in the two groups, and showed a trend towards higher values compared to the ApoE(-/-)/IKKe(-/-) mice, suggesting suppressive inflammatory activity in the heart of $\operatorname{ApoE}(-/-) / \operatorname{IKK} \varepsilon(-/-)$ mice. Thus, IKK $\varepsilon$ may contribute to the generation of low-grade, sustained inflammation in the heart after HFD feeding.

Ablation of IKKe blocks $N F-\kappa B$ activation induced by HFD. To investigate the regulatory mechanisms of IKKe in animal models, we examined the levels of p50, p65, IкB- $\alpha$ and the $\mathrm{NF}-\kappa \mathrm{B}$ pathway downstream factor (TGF- $\beta$, IL- $\beta$ ) proteins in hearts. By western blot analysis, the expression levels of total-p50 (T-p50) and total-p65 (T-p65), two important components that usually form the most common heterodimers of NF- $\kappa \mathrm{B}$, were shown not to be different between ApoE(-/-)/ $\mathrm{IKK \varepsilon (-/-)}$ and ApoE(-/-) murine groups (Fig. 4A). However, phosphorylated p50 (Pi-p50) and phosphorylated p65 (Pi-p65), comprising the functionally active form of the transcription factor in the nucleus, and I $\kappa \mathrm{B}-\alpha$, which binds with $\mathrm{NF}-\kappa \mathrm{B}$ to inhibit its activation, were also detected (Fig. 4A). The results showed that the expression of Pi-p50 and Pi-p65 (Fig. 4A) were significantly higher in the ApoE(-/-) group compared to the ApoE(-/-)/IKKe(-/-) group after 12 weeks HFD feeding, while the expression pattern of I $\mathrm{B}$ - $\alpha$ (Fig. 4A) was reversed among the four groups of mice. The protein expression of I $\mathrm{BB}-\alpha$ was highly increased in the HFD-fed ApoE(-/-)/IKKe(-/-) group compared to the ApoE(-/-) group, while that of the other two groups of mice remained at the same level. In addition, the protein expression of the NF- $\kappa \mathrm{B}$ pathway downstream factors, TGF- $\beta$ and IL- $\beta$, were also downregulated in the HFD-fed ApoE(-/-)/IKKe(-/-) group compared with the ApoE(-/-) group (Fig. 4B).

Consistent with results of the western blot analysis, we also observed that the expression levels of Pi-p50 and Pi-p65 were lower in the myocardium of the HFD-fed ApoE(-/-)/ 


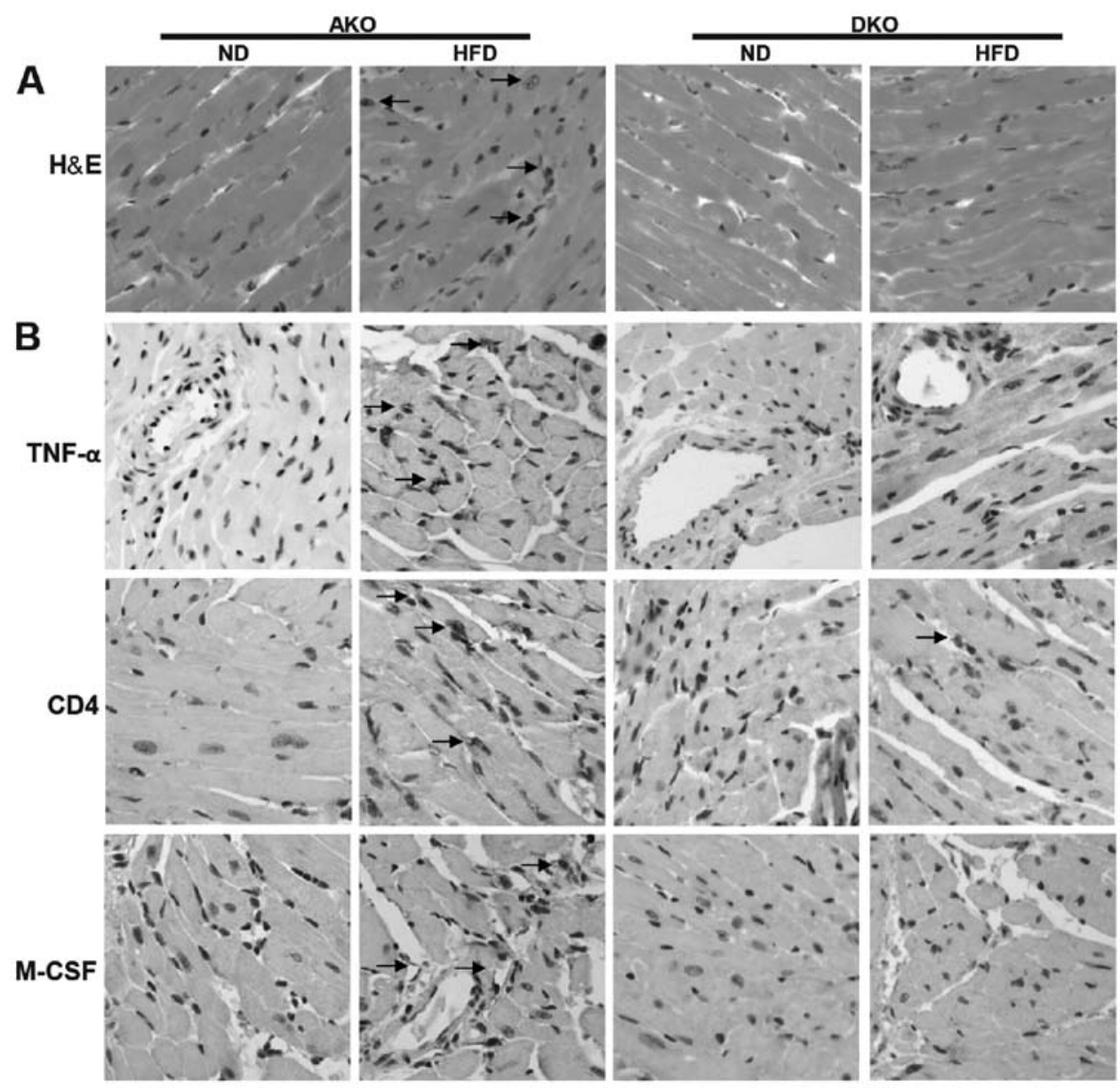

Figure 3. IKB kinase (IKK) $\varepsilon$ deficiency attenuates chronic, diet-induced inflammation in the heart after high-fat diet (HFD) feeding. (A) Hematoxylin and eosin (H\&E) staining of AKO and DKO mice fed with normal diet (ND) or HFD as indicated, $n=4$ per group. (B) Immunohistochemical analysis on the expression of inflammatory mediators including tumor necrosis factor- $\alpha$ (TNF- $\alpha$ ), CD4 T cells (CD4) and macrophage-colony stimulating factor (M-CSF) in the heart of AKO and DKO mice at 12 weeks fed with ND or HFD as indicated, $\mathrm{n}=4$ per group; magnification, $\mathrm{x} 400$.

$\operatorname{IKK\varepsilon (-/-)~group~than~the~ApoE(-/-)~group~by~immunofluores-~}$ cent analysis (Fig. 4C). Localization of Pi-p50 and Pi-p65 was widely distributed throughout the nucleus of the myocardium. The localization of these phosphorylated proteins transferred to the nuclei, suggesting that most of the activated forms of the nuclear factor shifted to the nuclei of the myocardium to function as a downstream target gene transcriptional regulator in the ApoE(-/-) group (Fig. 4D). Thus, these results confirm the inhibitory effect of IKK $\varepsilon$ ablation on $N F-\kappa B$ pathway.

IKKE-mediated inflammation is associated with the $N F-\kappa B$ pathway. To identify the molecular mechanisms underlying the positive effects of $\mathrm{IKK} \varepsilon$ on inflammatory response, we determined whether IKKe directly interacted with the T-p50/p65 heterodimer by immunoprecipitation experiments. The results showed that IKKe co-precipitated along with T-p50

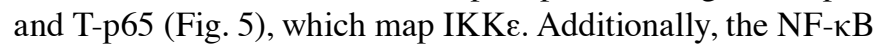
pathway mediated the interaction during inflammation. This interaction suggested that expression levels of the $\mathrm{NF}-\kappa \mathrm{B}$ pathway were significantly correlated with IKKe activation.

\section{Discussion}

Findings of previous studies suggest that obesity generates a state of chronic, low-grade inflammation $(15,22,23)$.
Transcription factor NF- $\kappa \mathrm{B}$ is a strong transcriptional activator that may play a crucial role in this response, although involvement of the liver and adipose tissue or whether the heart is affected and the processes involved, remain uncertain. The results of this study identify and delineate one pathway by which the non-canonical IKKe leads to the activation of canonical NF- $\kappa \mathrm{B}$ signaling, and plays a significant role in regulating the expression of genes involved in chronic, low-grade inflammatory response in hearts of obese mice.

In the present study, we first examined the mRNA levels of the inducible IKK family members induced by HFD in the ApoE(-/-) murine hearts. Notably, IKKe only was upregulated in the ApoE(-/-) mice-fed HFD. Consistent with this finding, the protein expression levels of IKKe in the hearts from the ApoE(-/-) mice after 12-week HFD also showed the same trends by western blot analyses. Furthermore, the monitoring of body weight showed that upregulation of IKKe may be correlated with weight gain, whereas the increased weight gain was completely blocked in the $\mathrm{ApoE}(-/-) / \mathrm{IKK} \varepsilon(-/-)$ group. Thus, these results suggest an inhibitory role for IKKe in HFD-induced obesity. Notably, under HFD, ablation of IKKe in mice did not significantly alter the total levels of plasma lipid levels, including TC, TG, LDL and HDL, which excluded the potential role for lipid alteration in the HFD-induced morphological and molecular adaptation. 

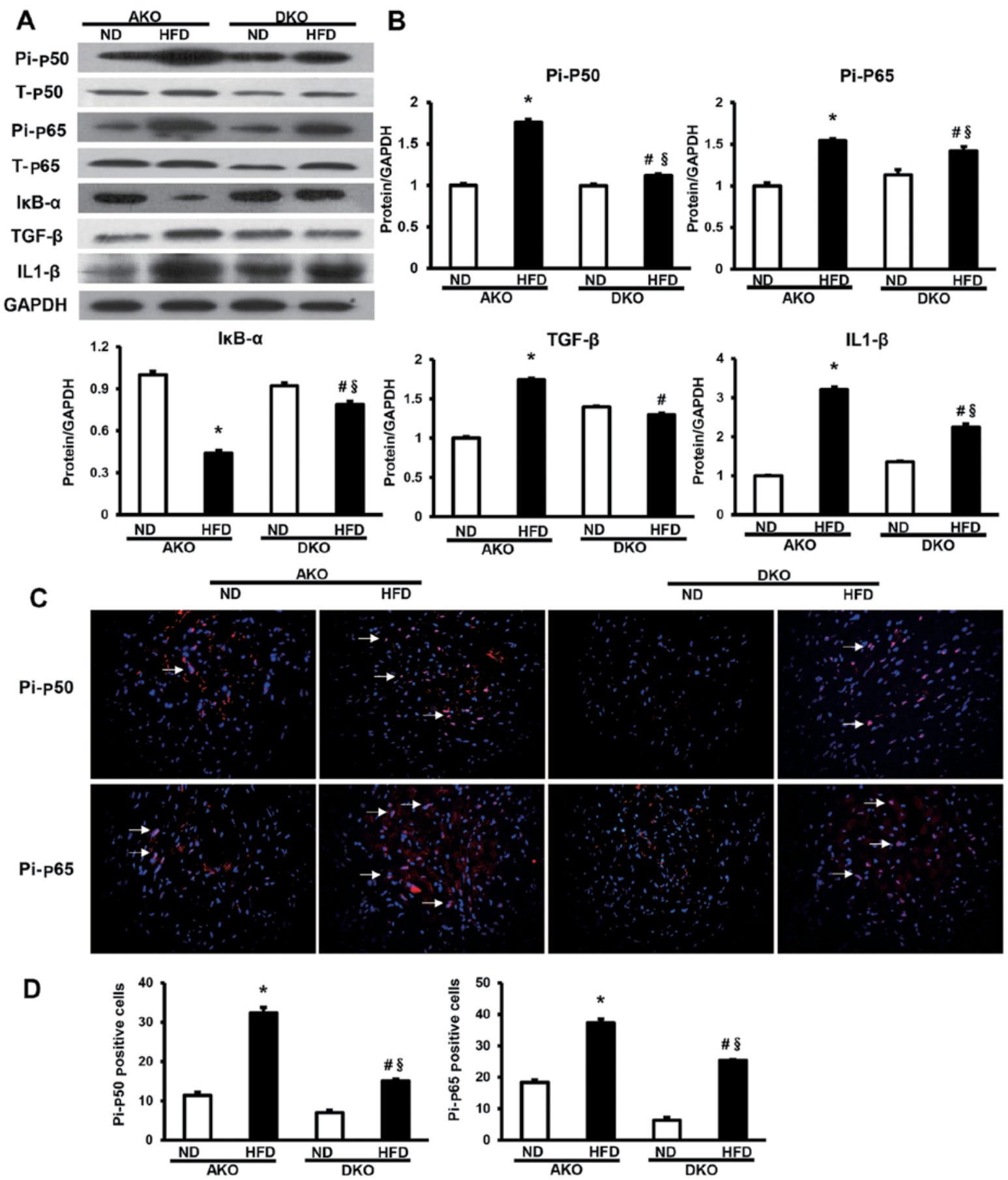

DKO
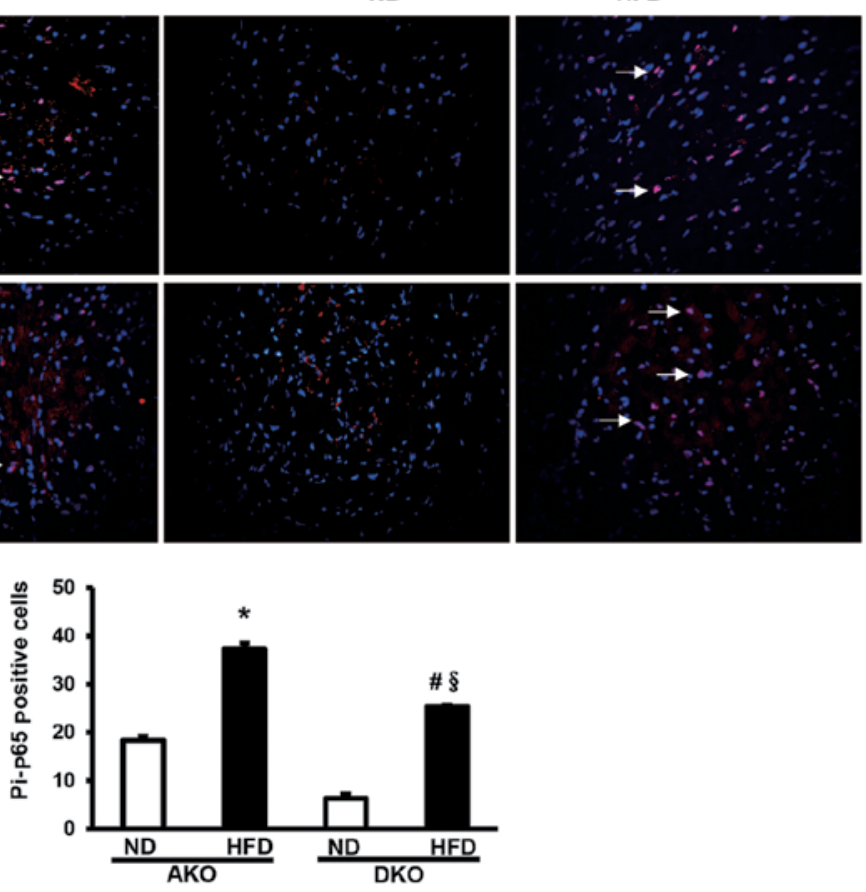

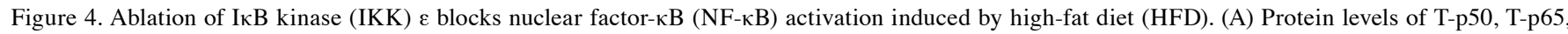
Pi-p50, Pi-p65, IкB- $\alpha$, TGF- $\beta$ and IL1- $\beta$ were measured by western blot analysis. (B) Quantitative results of the total-p50 (T-p50), total-p65 (T-p65), phosphorylated p50 (Pi-p50) and phosphorylated p65 (Pi-p65), IкB- $\alpha$, TGF- $\beta$ and IL1- $\beta$ in AKO and DKO murine hearts at 12 weeks fed with normal diet (ND) or HFD. Values are means \pm SEM, $n=4$ per group. ${ }^{*} \mathrm{P}<0.05$ vs. AKO ND; ${ }^{\circledR} \mathrm{P}<0.05$ vs. DKO ND; ${ }^{\text {P }}<0.05$ vs. AKO HFD; Densitometric data are from one representative experiment of three separate experiments. (C) Representative merged images show the protein expression of NF-kB cascade components Pi-p50 and Pi-p65 as determined by immunofluorescent staining. Nuclei (Hoechst, blue), positive staining (red), $n=4$ per group; magnification, $x 400$. (D) Localization of Pi-p50 and Pi-p65 was widely distributed throughout the nucleus of the myocardium.
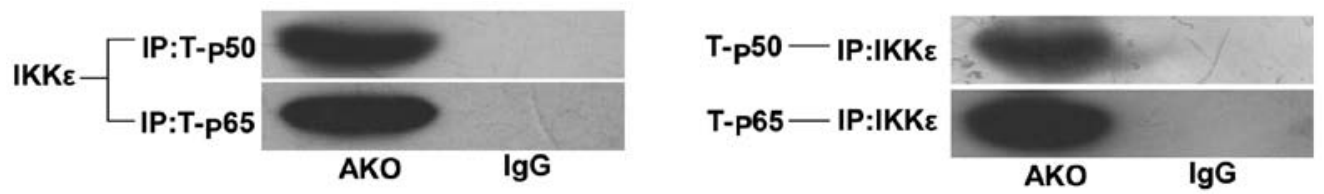

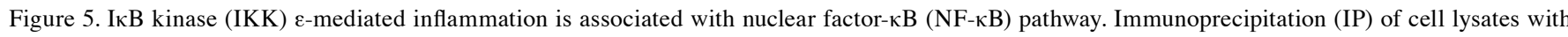
IKK $\varepsilon$ and immunoglobulin $\mathrm{G}(\mathrm{IgG})$ were analyzed by immunoblot (IB) analysis with total-p50 (T-p50) and total-p65 (T-p65). (B) Lysates IP with T-p50 and T-p65 and IgG were analyzed by IB with IKKe. Blots are representative of three independent experiments. 
To understand the effect of IKKe, which contributes to obesity-associated disorders, we examined the morphological and molecular alteration in hearts. A series of histopathological changes were evidenced by the application of H\&E staining, but not Oil Red O staining. In addition, under the HFD feeding regimen used here, we observed that the hearts of the $\operatorname{ApoE}(-/ /)$ mice had a low-grade, chronic inflammation/inflammatory cell infiltration, and certain inflammatory markers (TNF- $\alpha, \mathrm{CD} 4$, M-CSF) were elevated, which could be reversed by knockout of IKKe. However, whether IKK $\varepsilon$ is capable of facilitating obesity-induced inflammation remains controversial. At present, studies conducted by Scheja et al suggest that IKKe knockout had no effect on inflammation in major metabolic tissues and on insulin resistance when the anti-obesity effect of the IKK $\varepsilon$ knockout was overridden by a more aggressive HFD regimen (24). Thus, the inflammatory effects of IKK $\varepsilon$ in metabolic disease are apparently dependent on its obesogenic effects. Reilly et al study have also shown that the antiobesogenic effects of IKK $\varepsilon$ and TBK1 inhibitors, amlexanox, suppress inflammation and improve metabolic homeostasis in murine models of obesity (25). Thus, there is controversy regarding the effect of IKKe knockout on body weight regulation, inflammation and insulin resistance. As discussed above, it seems that multiple mechanisms are involved, but one of the first and potentially most important control mechanisms characterized for inflammation is its regulation of weight gain. The most significant metabolic effect of IKK $\varepsilon$ knockout in our study was also the prevention of weight gain on a HFD, which is consistent with recent advancements in the development of atherosclerosis from our laboratory (21). Thus, limitations of this study include the influence of the prevention of weight gain on inflammation. Concerning the cell, Olefsky et al showed that the protein kinase IKKe is an important connection between obesity and inflammation (26). As such, findings of this study show that IKKE is a bridge between obesity and inflammation $(21,26)$. Thus, the direct regulatory mechanism involving IKK $\varepsilon$ and inflammation in heart should be further investigated.

An essential component of the NF- $\mathrm{\kappa B}$ signaling mechanism is the canonical IKB kinase complex (IKK $\alpha$, IKK $\beta$ ), which is activated in response to many signals. The upstream activators and downstream target of this complex are well characterized (27). By contrast, relatively little is known concerning the related kinase IKKe. Another hypothesis in the present study was that the expression of IKKE would, at least partially, lead to HFD-inducing the activation of NF- $\mathrm{KB}$ cascade. The expression levels of Pi-p65/p50 in the murine hearts were all downregulated in the ApoE(-/-)/IKKe(-/-) group compared with the ApoE(-/-) group. Unlike the other components of the $\mathrm{NF}-\kappa \mathrm{B}$ cascade, the decrease in expression of IкB- $\alpha$ in the ApoE(-/-) group was reversed. This result may be explained by HFD inducing the NF- $\mathrm{KB}$ cascade through the phosphorylation

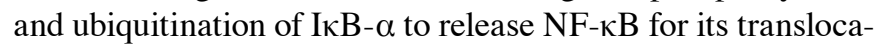
tion to the nucleus. Since there was more degradation of IкB- $\alpha$, the expression of the I $\mathrm{KB}-\alpha$ protein was likely to decrease, as confirmed by a previous study (27). Furthermore, consistent with the upstream NF- $\kappa \mathrm{B}$ components, our assessment of the protein levels of the two downstream inflammatory cytokine markers (IL1- $\beta$, TGF- $\beta$ ) exhibited the same trends in hearts. In this study, the expression and localization of the NF- $\kappa B$ cascade components were also investigated by immunofluorescence, which revealed that Pi-p65 and p50 were upregulated in the murine heart in the ApoE(-/-) group fed with a HFD, and suppressed its phosphorylation level and NF- $\mathrm{KB}$ signaling in the ApoE(-//)/IKKe(-/-) mice. Furthermore, we observed the translocation of resting NF- $\mathrm{KB}$ from the cytoplasm to the nuclei in the myocyte, consistent with the manner of NF- $\mathrm{KB}$ activation. Additionally, in the IP experiments, the inducible I $\mathrm{B}$ kinase of IKK $\varepsilon$ binds to the NF- $\kappa B$ cascade components total-p50 and total-p65, thereby providing a complete overview of the pathway, starting from the upstream IKKe to the downstream transcription factors. On the basis of our findings and those of previous studies suggesting this non-canonical

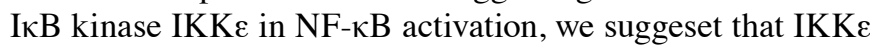
has a pivotal role in the activities of NF- $\mathrm{kB}$, which in turn regulates the expression of genes involved in the inflammatory response in hearts.

In conclusion, we have found a novel role for IKKe in regulating obesity-associated low-grade, chronic inflammation in murine hearts after HFD feeding, while in the absence of IKKE, the murine hearts are significantly prevented from sustaining an obesity-associated inflammatory response. These observations provide new insights that IKKe is dispensable for NF- $\kappa B$ activation and that IKK $\varepsilon$ may have anti-inflammatory properties in obesity.

\section{Acknowledgements}

This study was supported by grants from the National Natural Science Foundation of China $(81070180,81370259)$ and the Ministry of Human Resources and Social Security of China (2010-412).

\section{References}

1. Hotamisligil GS: Inflammation and metabolic disorders. Nature 444: 860-867, 2006.

2. Mauro $\mathrm{C}$ and Marelli-Berg FM: $\mathrm{T}$ cell immunity and cardiovascular metabolic disorders: does metabolism fuel inflammation? Front Immunol 3: 173, 2012.

3. Chen H: Cellular inflammatory responses: novel insights for obesity and insulin resistance. Pharmacol Res 53: 469-477, 2006.

4. Hansson GK and Hermansson A: The immune system in atherosclerosis. Nat Immunol 12: 204-212, 2011.

5. Harrison DG, Vinh A, Lob $\mathrm{H}$ and Madhur MS: Role of the adaptive immune system in hypertension. Curr Opin Pharmacol 10: 203-207, 2010.

6. Baker RG, Hayden MS and Ghosh S: NF- $\kappa B$, inflammation, and metabolic disease. Cell Metab 13: 11-22, 2011.

7. Ghosh S and Hayden MS: New regulators of NF-kappaB in inflammation. Nat Rev Immunol 8: 837-848, 2008.

8. Pasparakis M: Regulation of tissue homeostasis by NF-kappaB signalling: implications for inflammatory diseases. Nat Rev Immunol 9: 778-788, 2009.

9. Hayden MS and Ghosh S: Shared principles in NF-kappaB signaling. Cell 132: 344-362, 2008.

10. Oeckinghaus A, Hayden MS and Ghosh S: Crosstalk in NF- $\kappa B$ signaling pathways. Nat Immunol 12: 695-708, 2011.

11. Liu F, Xia Y, Parker AS and Verma IM: IKK biology. Immunol Rev 246: 239-253, 2012.

12. Wang N, Ahmed S and Haqqi TM: Genomic structure and functional characterization of the promoter region of human IkappaB kinase-related kinase IKKi/IKKvarepsilon gene. Gene 353: 118-133, 2005.

13. Häcker $H$ and Karin M: Regulation and function of IKK and IKK-related kinases. Sci STKE 2006: re13, 2006.

14. Chau TL, Gioia R, Gatot JS, et al: Are the IKKs and IKK-related kinases TBK1 and IKK-epsilon similarly activated? Trends Biochem Sci 33: 171-180, 2008. 
15. Chiang $\mathrm{SH}$, Bazuine $\mathrm{M}$, Lumeng $\mathrm{CN}$, et al: The protein kinase IKKepsilon regulates energy balance in obese mice. Cell 138: 961-975, 2009

16. Dai J, Shen DF, Bian ZY, et al: IKKi deficiency promotes pressure overload-induced cardiac hypertrophy and fibrosis. PloS One 8: e53412, 2013.

17. Guo J, Kim D, Gao J, et al: IKBKE is induced by STAT3 and tobacco carcinogen and determines chemosensitivity in non-small cell lung cancer. Oncogene 32: 151-159, 2013.

18. Guo JP, Coppola D and Cheng JQ: IKBKE protein activates Akt independent of phosphatidylinositol 3-kinase/PDK1/mTORC2 and the pleckstrin homology domain to sustain malignant transformation. J Biol Chem 286: 37389-37398, 2011

19. Tenoever BR, Ng SL, Chua MA, McWhirter SM, García-Sastre A and Maniatis T: Multiple functions of the IKK-related kinase IKKepsilon in interferon-mediated antiviral immunity. Science 315: 1274-1278, 2007.

20. Ng SL, Friedman BA, Schmid S, et al: IкB kinase epsilon (IKK(epsilon)) regulates the balance between type I and type II interferon responses. Proc Natl Acad Sci USA 108: 21170-21175, 2011.
21. Cao C, Zhu Y, Chen W, et al: IKKe knockout prevents high fat diet induced arterial atherosclerosis and NF- $\kappa \mathrm{B}$ signaling in mice. PloS One 8: e64930, 2013.

22. Shoelson SE, Herrero L and Naaz A: Obesity, inflammation, and insulin resistance. Gastroenterology 132: 2169-2180, 2007.

23. Wellen KE and Hotamisligil GS: Inflammation, stress, and diabetes. J Clin Invest 115: 1111-1119, 2005.

24. Scheja L, Heese B and Seedorf K: Beneficial effects of IKKe-deficiency on body weight and insulin sensitivity are lost in high fat diet-induced obesity in mice. Biochem Biophys Res Commun 407: 288-294, 2011.

25. Reilly SM, Chiang SH, Decker SJ, et al: An inhibitor of the protein kinases TBK1 and IKK- $\varepsilon$ improves obesity-related metabolic dysfunctions in mice. Nat Med 19: 313-321, 2013.

26. Olefsky JM: IKKepsilon: a bridge between obesity and inflammation. Cell 138: 834-836, 2009.

27. Karin $M$ and Ben-Neriah Y: Phosphorylation meets ubiquitination: the control of NF-[kappa]B activity. Annu Rev Immunol 18: 621-663, 2000. 